\title{
miRNA and nasopharyngeal carcinoma
}

\author{
ZHAO LuQing ${ }^{1,2,3^{*}}$, CHEN Xue ${ }^{1,2,3} \&$ CAO Ya $a^{1,2,3}$ \\ ${ }^{1}$ Key Laboratory of Carcinogenesis and Invasion, Ministry of Education, Changsha 410078, China; \\ ${ }^{2}$ Key Laboratory of Carcinogenesis, Ministry of Health, Changsha 410078, China; \\ ${ }^{3}$ Cancer Research Institute, Xiangya School of Medicine, Central South University, Changsha 410078, China
}

Received October 12, 2010; accepted December 15, 2010

\begin{abstract}
MicroRNAs (miRNAs) constitute a family of small non-coding RNA molecules 22-25 nucleotides in length. miRNAs control the expression of target genes at the post-transcriptional level by inhibiting translation or by degrading target mRNA through binding to complementary sequences in the 3'-untranslated regions. Recent studies have demonstrated that miRNAs are intimately involved in processes leading to nasopharyngeal carcinoma, such as the Epstein-Barr virus-encoded latent membrane protein 1 activated signal transduction pathways, gene-regulatory networks, mitosis, tumor angiogenesis, invasion and migration. Exploring the relationship between miRNAs and the development of nasopharyngeal carcinoma, will further the understanding of this cancer and provide new avenues for diagnosis and treatment.
\end{abstract}

miRNA, nasopharyngeal carcinoma, Epstein-Barr virus, latent membrane protein 1, signaling transduction pathway, mitosis, invasion, migration

Citation: Zhao L Q, Chen X, Cao Y. miRNA and nasopharyngeal carcinoma. Chinese Sci Bull, 2011, 56: 722-728, doi: 10.1007/s11434-010-4330-x

MicroRNAs (miRNAs) have been intensively studied in recent years. Although consisting of only 22-25 nucleotides, miRNAs exert crucial biological functions. miRNA expression varies in different kinds of tumor tissues and they have been shown to be involved in the initiation and progression of tumors [1].

Nasopharyngeal carcinoma (NPC) is a head and neck epithelial malignancy that occurs frequently in Southern China [2]. It is characterized by high invasiveness and metastasis, and involves Epstein-Barr virus (EBV) infection and numerous genetic factors [3]. In this review, we focus on recent studies [4] that have described miRNA involvement in the carcinogenesis of NPC.

\section{1 miRNA biogenesis, regulatory mechanisms and biological functions}

\section{1 miRNA biogenesis and regulatory mechanisms}

miRNAs are small non-coding RNA molecules of about

*Corresponding author (email: Lqz2010@hotmail.com; xiaoqing1213@sina.com)
22-25 nucleotides in length. miRNA genes are first transcribed by RNA polymerase II, to produce molecules hundreds of nucleotides in length, called pri-miRNAs. Then, Drosha, an RNA polymerase III, processes pri-miRNAs into stem-loop structures, 60-70 nucleotides in length, called pre-miRNAs. The transportation protein, Exportin-5, identifies and combines with the 3 '-end of pre-miRNAs and, in a Ran-GTP-dependent manner, pre-miRNAs are exported from the nucleus into cytoplasm. With the involvement of another RNase III Dicer, the pre-miRNAs are further cleaved into double stranded RNAs (dsRNAs). A helicase then degrades one strand of the dsRNA, leaving the other strand a mature active miRNA to enter the nucleus and form the miRNA-associated RNA-induced silencing complex (miRISC) [5-7]. Within this complex, miRNAs can play essential roles in biological functions.

miRNAs control the expression of target genes at the post-transcriptional level by inhibiting protein translation or by degrading cognate target mRNAs through binding to their 3'-untranslated regions (UTRs) with varying degrees of sequence complementarity. miRNAs are also involved in 
post-transcriptional regulation and multiple biological functions [8,9]. Most plant miRNAs are 100\% complementary with their target genes and fully degrade their target mRNAs; however, miRNAs in animals often have only partial complementarity with target genes, so post-transcriptional translation is inhibited rather than completely blocked [10]. As many miRNAs are not completely complementary with target mRNAs, post-transcriptional repression is the main mode of regulation [11]. Because one miRNA can target various mRNAs and one mRNA can be targeted by several miRNAs, this small regulator and target genes constitute a complicated regulation network and adds a new layer of complexity to the control of gene expression [12].

\subsection{Biological functions of miRNA}

The target genes of miRNAs are involved in diverse biological functions, including the development, differentiation, proliferation, apoptosis, stress response and maintenance of stem cell differentiation potential [13]. miRNAs are also highly important in the regulation of many biological processes involved in carcinogenesis, such as tumor cell proliferation, apoptosis, adhesion and tumor angiogenesis. Approximately $50 \%$ of miRNAs are located in cancerrelated genome regions, which have a wide and vital role in the genetic level control of carcinogenesis [14]. Many of the miRNAs act as oncogenes or tumor suppressors $[15,16]$. miRNAs that have oncogene capability are usually called "oncomiRs" and include miR-155, miR-17-92 cluster (miR-17-5p, miR-17-3p, miR-18a, miR-19a, miR-20a, miR-19a-1, miR-92-1) and miR-21 [17-19]. In contrast, the tumor suppressor miRNAs include miR-15a, miR-16-1, let-7 family, miR-143, miR-145 and miR-34a [20]. Douglas [21] called the miRNAs involved in metastasis "metastamir"; those that promote metastasis include miR-10b, miR-21, and those that inhibit metastasis include miR-141, $\mathrm{miR}-200 \mathrm{a} / \mathrm{b} / \mathrm{c}, \mathrm{miR}-146$ and $\mathrm{miR}-335$.

\section{Involvement of miRNA in the molecular bi- ology of nasopharyngeal carcinoma}

\section{1 miRNA and EBV}

The $E B V$ could encode multiple miRNA genes, and it was the first virus shown to encode miRNA genes [22]. According to recent data provided by miRBase [23], the $E B V$-encoded miRNAs include ebv-miR-BHRF1-1 3 and ebv-miR-BART1 22. They are located at separate loci in the virus genome, at BHRF1 and BART [24]. BHRF1 is an early gene of virus replication, which is highly expressed when the lytic and duplicated level of virus is high. It is involved in the regulation of lytic and replication cycles [25]. BART is expressed during the latent cycle, and inhibits the replication of virus, keeping the duplicate level low [26].
$E B V$-encoded miRNAs are involved in the regulation of the lytic-latent switch of EBV and in the resistance to host immune responses [27]. These miRNAs not only target the virus' own genes, but also target the host genes [28]. Xia et al. [29] reported that miR-BHRF1-3 targets the host gene, CXCL11, the repression of which will protect EBV-infected $\mathrm{B}$ cells from attack by cytotoxic T cells. Choy et al. [30] also demonstrated that miR-BART5 targets the host gene, PUMA (p53-upregulated modulator of apoptosis), the down-regulation of which will inhibit apoptosis of the virus infected host cells.

Iizasa et al. [31] found that the primary transcripts of ebv-miR-BART6 (pri-miR-BART6) are edited in latent EBV-infected cells, and that mature miR-BART6 is essential for various stages of latency in cells. Further study [31] showed that miR-BART6-5p RNAs could suppress the expression of the EBNA2 viral oncogene, which is required for the transition from type I and II latency (less responsive to the immune system) to type III latency (more active to the immune system). Also, Zta and Rta viral proteins are essential for lytic replication of EBV [32]. These results revealed that miR-BART6 plays a critical role in the regulation of EBV infection and latency.

These studies indicate that the $E B V$-encoded miRNAs play a vital role in the development of NPC. They not only modulate the virus' own genes to facilitate virus infection and latency, but they also affect host target genes to help the virus escape from the host immune responses and inhibit apoptosis of host cells.

\section{$2.2 \operatorname{miRNA}$ and $E B V$-encoded LMP1}

$E B V$-encoded LMP1 (latent membrane protein 1) is an important viral oncoprotein in NPC development [33]. LMP1 is produced during EBV type II and III latency, and controls the NF- $\kappa \mathrm{B}$ signaling pathway and the growth and apoptosis of host cells. Natalie et al. [34] showed that LMP1 activates the miR-146a promoter through an NF- $\kappa \mathrm{B}$ dependent pathway and induces the expression of miR-146a in B-lymphocytes. miR-146a also plays a role in the induction or maintenance of EBV latency by modulating innate immune responses to virus-infected host cells [35]. Graziana et al. [36] also demonstrated that LMP1 trans-activates miR-155 transcription through activating an NF- $\kappa \mathrm{B}$ pathway, which plays a critical role in the activation of lymphocytes and in the EBV-mediated transformation of B cells. PU.1 is the downstream target gene of miR-155, which is highly expressed in miR-155-deficient B cells and leads to reduced numbers of IgG1-switched B cells [36]. Together, these observations indicate that miR-155 has a role in antigen-driven B cell maturation.

Lo et al. [37] reported that there are three $E B V$-encoded miRNAs, miR-BART1-5p, miR-BART16 and miRBART17$5 \mathrm{p}$, which can downregulate the expression of LMP1. Zheng et al. [38] suggested that LMP1 triggers the NF- $\mathrm{B}, \mathrm{AP}-1$ 
and STAT signaling pathways; moreover, the JAK/STAT, PI-PLC-PKC pathways become active by upregulating the expression of JAK3 (Janus kinase 3) and enhancing the phosphorylation of STAT (signal transducers and activators of transcription); the constitutive activation of signaling cascades triggered by LMP1 can induce a diverse array of biological effects that ultimately lead to cell cycle disruption, the acceleration of G1/S phase and the arrest of G2/M phase; LMP1 can also induce the expression of hTERT (human telomerase reverse transcriptase) and promote cell immortalization.

These findings reveal that $E B V$-encoded miRNAs can regulate the expression of LMP1. At the same time, LMP1 can activate signal transduction pathways that activate the expression of miRNAs, which then regulate tumor-related target genes, ultimately lead to the development of NPC.

\section{3 miRNA in signal transduction pathways and in gene-regulatory networks of NPC}

Recent studies [39] suggest that multiple miRNAs may work in concert to regulate related target genes in a common pathway. Therefore, the study of signal transduction pathways rather than individual target genes will provide new insight into the biological functions of miRNA. Chen et al. [40] recently reported that there are 35 miRNAs whose expression levels are significantly changed in NPC tissue biopsies (11 miRNAs, including miR-196b, miR-138, miR-155, miR-142-3p and miR-18a are upregulated in NPC samples; 24 miRNAs, including miR-204, miR-449a, miR-34c-3p, miR-143 and miR-145 are downregulated in NPC samples). Among the 35 miRNAs, there are four miRNAs (miR-142-3p, miR-196b, miR-138 and miR-155) whose expression levels are significantly upregulated and another four (miR-204, miR-195, miR-187 and miR-143) whose expression levels are significantly downregulated. There are five specific pathways targeted by downregulated miRNAs: (1) regulation of the G1/S transition; (2) cross-talk between VEGF (vascular endothelial growth factor) and angiopoietin-1 [41,42] signaling; (3) G-protein(-) mediated regulation of MARK-ERK signaling; (4) TGF (transforming growth factor), WNT [43] and cytoskeletal remodeling; (5) signaling by interaction of membrane-bound ESR1 (estrogen receptor 1) with G-proteins. Among these five pathways, regulation of the G1/S transition and cross-talk between VEGF and angiopoietin-1 signaling are the two most relevant pathways for NPC targeted by miRNAs [40].

Further discoveries [40] confirmed that the expression levels of six target genes (CCND2, CCND3, CDC25A, VEGFA, PLCG1 and AKT) are upregulated in the two most significant pathways targeted by downregulated miRNAs. In other words, the predicted target genes of downregulated miRNAs are upregulated in NPC. The studies [40] described above indicate that the signaling pathways targeted by downregulated miRNAs are involved in the regulation of cell cycle, cell survival, apoptosis, and cytoskeletal remodeling. Together, these results suggest that the upregulation of a predicted target gene has an inverse correlation with the downregulation of a specific miRNA.

miRNAs together with oncogenes, tumor suppressor genes and related target genes form a complex tumorigenic network in the carcinogenesis of NPC. Zhang et al. [44] showed that miR-141 is upregulated in NPC, and acts as an oncogene. The expression level of miR-141 can be downregulated by knocking down the oncogene $c-M Y C$ and reexpressing tumor suppressor gene, SPLUNC1. The downregulation of miR-141 can affect cell cycle, cell growth, apoptosis, migration and metastasis. Using luciferase reporter assays and Western blot analysis, Zhang et al. [44] further confirmed that $B R D 3, U B A P 1$ and PTEN are potential targets of miR-141. BRD3 and UBAP1 [45-47] are both involved in the development of NPC. BRD3 participates in the regulation of the $\mathrm{Rb} / \mathrm{E} 2 \mathrm{~F}$ pathway. PTEN participates in the regulation of the AKT signaling pathway and acts as a vital tumor suppressor gene in various cancers [48]. Inhibition of miR-141 expression affects the biological functions of various molecules in Rb/E2F, JNK2 and AKT signaling pathways [44]. Zhang et al. [44] suggested that miR-141 and tumor-related genes, $c-M Y C, S P L U N C 1, B R D 3, U B A P 1$ and PTEN are all involved in a "gene-miRNA" network, which ultimately leads to the development of NPC.

Taken together, these studies indicate that during the development of NPC, a series of genetic and epigenetic events constitute a complicated network system [49-53]. Both oncogenes and tumor suppressor genes can regulate the expression of miRNAs; meanwhile, the expression of the relevant miRNA could further modulate the expression of tumor-related target genes. Hence, miRNAs, oncogenes, tumor suppressor genes and tumor-related target genes are all involved in a complex "gene-miRNA-gene" network system, which ultimately contributes to the progression of NPC.

\section{Role of miRNA in the cell biology of naso- pharyngeal carcinoma}

\section{1 miRNA and mitosis}

Plk1 is a vital regulator in many stages of mitosis and also plays a critical role in the cellular proliferation and progression of NPC [54]. The abnormal expression of Plk1 correlates with the downregulation of miRNA [55]. Plk1, which is a vital regulator in the $\mathrm{G} 2 / \mathrm{M}$ transition of mitosis, is essential for cell cycle regulation [56]. Wei et al. [55] found that miR-100 can modulate the expression of Plk1 in NPC; siPlk1, which is a siRNA targeted to Plk1, could alter cell structures and induce aberrant spindle formation; Plk1 depletion can also induce abnormal microtubule formation and lead to the condensation of chromatin in cancer cells [57]. 
Wei et al. [55] further showed that siPlk1 could inhibit the expression of Plk1 mRNA and protein; the combination of ionizing radiation and siPlk1 effectively reduces the viability of C666- 1 cells and increases $\gamma \mathrm{H} 2 \mathrm{AX}$ levels. Plk1 depletion induces significant $\mathrm{G} 2 / \mathrm{M}$ arrest and apoptosis mediated by caspase $3 / 7$ activation [58]. Radiation enhances G2/M arrest and apoptosis [59], so the combination of radiation and siPlk1 may delay tumor formation [60].

Hence, the underexpression of miR-100 will lead to the overexpression of Plk1, which in turn contributes to NPC development. So targeting of Plk1 to inhibit its overexpression will promote mitotic catastrophe and cell cycle arrest. This strategy has the potential to become an extremely powerful therapeutic approach when combined with effective ionizing radiation $[61,62]$.

\section{2 miRNA and tumor angiogenesis, invasion and mi- gration}

Angiogenesis is fundamental for a wide variety of physiological and pathological processes, including development, wound healing, inflammation and tumor formation [63]. Many small molecules play a positive role in the regulation of angiogenesis. Among them, vascular endothelial growth factor (VEGF) is the most important and its expression is under the control of multiple factors [64]. Hua et al. [65] determined that miR-16, miR-20a, miR-20b, let-7b, miR-17-5p, miR-27a, miR-106a, miR-106b, miR-107, miR-193a, miR-210, miR-320, miR-361 and miR-15b are predicted to target VEGF, and they may all modulate the expression of VEGF.

Olsson et al. [66] demonstrated that under hypoxia stimulation, VEGF and other angiogenic factors are upregulated; these factors work together to induce angiogenesis; under strict spatio-temporal conditions, miRNAs and various target genes can co-regulate multiple functionally related genes and angiogenic factors. Hua et al. [65] further showed that miR-15b, miR-16, miR-20a and miR-20b all have an effect on angiogenic factors in NPC cells; at the same time, there are seven angiogenesis related genes, VEGF, c-MET, COX2, uPAR, PAII, MAPK7 and Ang that are all upregulated in NPC cells.

$\mathrm{Li}$ et al. [67] reported that LMP1, which acts as a key regulator in promoting NPC metastasis [68], may induce the expression of transcription factor Twist, which in turn upregulates the expression of miR-10b to contribute to the metastasis of NPC. Meanwhile, by activating various signal transduction pathways, LMP1 can regulate the expression levels of multiple metastasis related genes, such as E-cadherin, MMPs, c-Met, VEGF, EGFR and COX-2 [69].

Sikumar et al. [70] suggested that the under-expression of miR-29c in NPC will lead to the upregulation of downstream target genes, which encode multiple extracellular matrix proteins, including collagens and laminin $\gamma 1$ that are associated with invasion and migration of NPC cells. Hence, the downregulation of miR-29c in NPC cells contributes to the invasive and metastatic characteristics of this cancer. Xia et al. [71] determined that miR-200a is downregulated and mediates the downregulation of the target genes, ZEB2 and CTNNB1, which inhibit cell growth, migration and metastasis in NPC.

These studies suggest that different kinds of miRNA and their target genes are closely related to angiogenesis, invasion and migration of NPC cells [72]. The interactions of miRNA and target genes could contribute to the metastasis of NPC. How can we modulate the expression of metastasis-related miRNAs? And how can we block the biological functions of target genes? Answers to these questions require further research.

\section{Multiple NPC-related miRNAs and future research}

From the studies described above, we list the relationships between miRNA and NPC (Table 1) and summarize the recent discoveries related to NPC and miRNAs.

From Table 1, we can see that there is great interest in studying the relationships between miRNA and the carcinogenesis of NPC. The fields of study range from $E B V$-encoded miRNAs, which affect host biological functions, to the host cell's self-encoded miRNAs, which play a crucial role in the development of NPC. Furthermore, we recommend that additional studies of miRNA are justified to answer questions such as: Do miRNAs exist in NPC that can enter the nucleus and function as transcription factors? Is there an RNA binding protein, similar to Dnd1 (Dead end 1 ), that could bind to the adjacent genomic regions where miRNAs and target genes interact with each other? Can such an RNA binding protein indirectly inhibit the interactions between miRNA and target genes? In NPC diagnosis and treatment, does a highly specific miRNA exist that can be detected in serum or body fluid of NPC patients? Can this miRNA act as a biomarker for the early diagnosis of NPC? In addition, for chemotherapy or radiation therapy, do miRNAs exist whose expression would enhance the sensitivity of treatment? These problems are critical issues that need to be addressed in future work.

Recent progress in understanding NPC carcinogenesis has shown that miRNAs are involved in LMP1 signaling. Future work should, therefore, focus on exploring the interactions between miRNAs and kinases, transcription factors and target genes in the signal transduction pathways activated by LMP1. We should also pay more attention to the metastasis-related genes. LMP1 can modulate the expression of miRNAs that then regulate the expression of metastasis-related genes; therefore, addressing these areas will help produce a better understanding of the role of miRNA in the invasion and 
Table 1 NPC-related miRNA ${ }^{\text {a) }}$

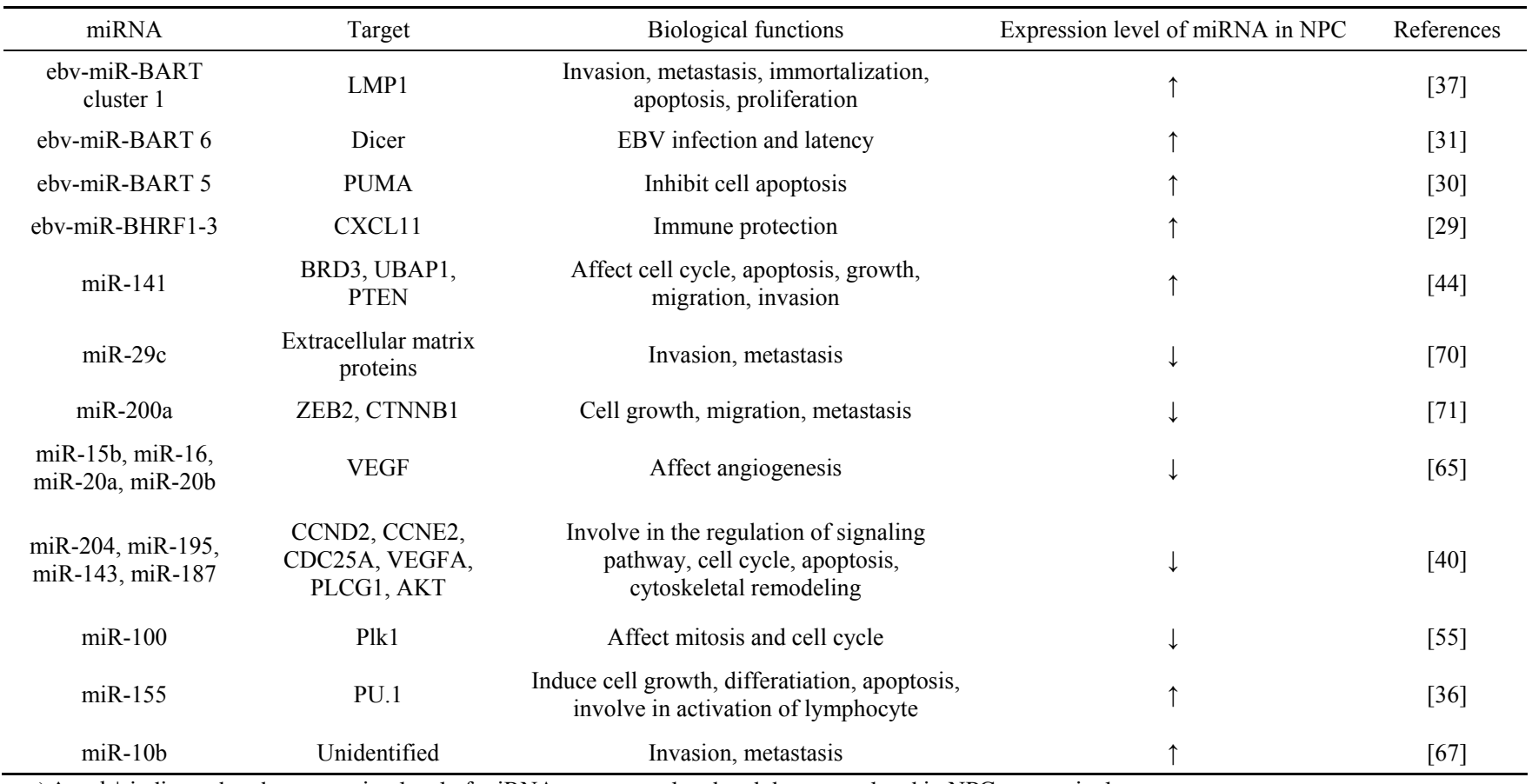

a) $\uparrow$ and $\downarrow$ indicate that the expression level of miRNA are up-regulated and down-regulated in NPC, respectively.

metastasis of NPC, and will also provide new targets for the treatment of metastasis and recurrence of NPC.

$\mathrm{Plk} 1$ is another target that warrants attention. Plk1 has an important role in the regulation of the cell cycle and mitosis in NPC, at the same time, interacts with the effects of radiation therapy. Therefore, further research on the interaction between Plk1 related miRNAs and radiation will bring new hope for the treatment of NPC.

In conclusion, we believe that further research on the relationships between miRNA and NPC carcinogenesis will not only provide new understanding of the development of NPC, but also provide new avenues for the diagnosis and treatment of NPC.

This work was supported by the National Key Basic Research Program of China (2011CB504300) and the Key Project of National Natural Science Foundation of China (30930101).

1 George A C, Carlo M C. MicroRNA signatures in human cancers. Nat Rev Cancer, 2006, 6: 857-866

2 Chin D, Boyle G M, Porceddu S, et al. Head and neck cancer: Past, present and future. Expert Rev Anticancer Ther, 2006, 6: 1111-1118

3 Deyrup A T. Epstein-Barr virus-associated epithelial and mesenchymal neoplasms. Hum Pathol, 2008, 39: 473-483

4 Zhu J Y, Pfuhl T, Motsch N, et al. Identification of novel Epstein-Barr virus microRNA genes from nasopharyngeal carcinomas. $\mathrm{J}$ Virol, 2009, 83: 3333-3341

5 Georgia S. Emerging roles of microRNAs as molecular switches in the integrated circuit of the cancer cell. RNA, 2009, 15: 1443-1461

6 Filipowicz W, Bhattacharyya S N, Sonenberg N. Mechanisms of post-transcriptional regulation by microRNAs: Are the answers in sight? Nat Rev Genet, 2008, 9: 102-114
7 Israel A, Sharan R, Ruppin E, et al. Increased microRNA activity in human cancers. PLoS One, 2009, 4: e6045

8 Olson $\mathrm{P}$, Lu J, Zhang $\mathrm{H}$, et al. MicroRNA dynamics in the stages of tumorigenesis correlate with hallmark capabilities of cancer. Genes Dev, 2009, 23: 2152-2165

9 Zhang B, Pan X, Cobb G P, et al. MicroRNAs as oncogenes and tumor suppressors. Dev Biol, 2007, 302: 1-12

$10 \mathrm{Wu}$ M, Jolicoeur N, Li Z, et al. Genetic variations of microRNAs in human cancer and their effects on the expression of miRNAs. Carcinogenesis, 2008, 29: 1710-1716

11 Kumar M S, Lu J, Mercer K L, et al. Impaired microRNA processing enhances cellular transformation and tumorigenesis. Nature Genet, 2007, 39: 673-677

12 Liu C G, Calin G A, Volinia S, et al. MicroRNA expression profiling using microarrays. Nat Protoc, 2008, 3: 563-578

13 Hatfield S, Ruohola-Baker H. MicroRNA and stem cell function. Cell Tissue Res, 2008, 331: 57-66

14 Bushati N, Cohen S M. MicroRNA functions. Annu Rev Cell Dev Biol, 2007, 23: 175-205

15 Finoux A L, Chartrand P. Oncogenic and tumour suppressor microRNAs. Med Sci (Paris), 2008, 24: 1049-1054

16 Wang X, Tang S, Le S Y, et al. Aberrant expression of oncogenic and tumor-suppressive microRNAs in cervical cancer is required for cancer cell growth. PLoS One, 2008, 3: e2557

17 Aurora E K, Frank J S. Oncomirs-microRNAs with a role in cancer. Nat Rev Cancer, 2006, 6: 259-269

18 Rai D, Karanti S, Jung I, et al. Coordinated expression of microRNA-155 and predicted target genes in diffuse large B-cell lymphoma. Cancer Genet Cytogenet, 2008, 181: 8-15

19 Takakura S, Mitsutake N, Nakashima M, et al. Oncogenic role of miR-17-92 cluster in anaplastic thyroid cancer cells. Cancer Sci, 2008, 99: 1147-1154

20 Welch C, Chen Y, Stallings R L. MicroRNA-34a functions as a potential tumor suppressor by inducing apoptosis in neuroblastoma cells. Oncogene, 2007, 26: 5017-5022

21 Douglas R H. Metastamir: The field of metastasis-regulatory microRNA is spreading. Cancer Res, 2009, 69: 7495-7498

22 Cosmopoulos K, Pegtel M, Hawkins J, et al. Comprehensive 
profiling of Epstein-Barr virus microRNAs in nasopharyngeal carcinoma. J Virol, 2009, 83: 2357-2367

23 Griffiths-Jones S, Saini H K, van Dongen S, et al. miRBase: Tools for microRNA genomics. Nucleic Acids Res, 2008, 36: D154-D158

24 Gourzones C, Gelin A, Bombik I, et al. Extra-cellular release and blood diffusion of BART viral micro-RNAs produced by EBVinfected nasopharyngeal carcinoma cells. Virol J, 2010, 7: 271

25 Jing Y Z, Wang Y, Jia Y P, et al. Polymorphisms of Epstein-Barr virus BHRF1 gene, a homologue of bcl-2. Chin J Cancer, 2010, 29: 1000-1005

26 Edwards R H, Marquitz A R, Raab-Traub N. Epstein-Barr virus BART microRNAs are produced from a large intron prior to splicing. J Virol, 2008, 82: 9094-9106

27 Ghosh Z, Mallick B, Chakrabarti J. Cellular versus viral microRNAs in host-virus interaction. Nucleic Acids Res, 2009, 37: 1035-1048

28 Cullen B R. Viral and cellular messenger RNA targets of viral microRNAs. Nature, 2009, 457: 421-425

29 Xia T, O'Hara A, Araujo I, et al. EBV microRNAs in primary lymphomas and targeting of CXCL-11 by ebv-mir-BHRF1-3. Cancer Res, 2008, 68: 1436-1442

30 Choy E Y, Siu K L, Kok K H, et al. An Epstein-Barr virus-encoded microRNA targets PUMA to promote host cell survival. J Exp Med, 2008, 205: 2551-2560

31 Iizasa H, Wulff B E, Alla N R, et al. Editing of Epstein-Barr virus-encoded BART6 microRNAs controls their dicer targeting and consequently affects viral latency. J Biol Chem, 2010, 285: 33358-33370

32 Chang L K, Chuang J Y, Nakao M, et al. MCAF1 and synergistic activation of the transcription of Epstein-Barr virus lytic genes by Rta and Zta. Nucleic Acids Res, 2010, 38: 4687-4700

33 Hariwiyanto B, Sastrowiyoto S, Mubarika S, et al. LMP1 and LMP2 may be prognostic factors for outcome of therapy in nasopharyngeal cancers in Indonesia. Asian Pac J Cancer Prev, 2010, 11: 763-766

34 Natalie M, Thorsten P, Jan M, et al. Epstein-Barr virus-encoded latent membrane protein 1 (LMP1) induces the expression of the cellular microRNA miR-146a. RNA Biol, 2007, 4: 131-137

35 Middeldorp J M, Pegtel D M. Multiple roles of LMP1 in Epstein-Barr virus induced immune escape. Semin Cancer Biol, 2008, 18: 388-396

36 Graziana G, Annalisa R, Daniela R, et al. Epstein-Barr virus latent membrane protein 1 trans-activates miR-155 transcription through the NF- $\kappa$ B pathway. Nucleic Acids Res, 2008, 36: 6608-6619

37 Lo A K, To K F, Lo K W, et al. Modulation of LMP1 protein expression by $E B V$-encoded microRNAs. Proc Natl Acad Sci USA, 2007, 104: 16164-16169

38 Zheng H, Li L L, Hu D S, et al. Role of Epstein-Barr virus encoded latent membrane protein 1 in the carcinogenesis of nasopharyngeal carcinoma. Cell Mol Immunol, 2007, 4: 185-196

39 Liu Q, Fu H, Sun F, et al. miR-16 family induces cell cycle arrest by regulating multiple cell cycle genes. Nucleic Acids Res, 2008, 36: 5391-5404

40 Chen H C, Chen G H, Chen Y H, et al. MicroRNA deregulation and pathway alterations in nasopharyngeal carcinoma. Br J Cancer, 2009, 100: 1002-1011

$41 \mathrm{Li} \mathrm{Y} \mathrm{H,} \mathrm{Hu} \mathrm{C} \mathrm{F,} \mathrm{Shao} \mathrm{Q,} \mathrm{et} \mathrm{al.} \mathrm{Elevated} \mathrm{expressions} \mathrm{of} \mathrm{survivin} \mathrm{and}$ VEGF protein are strong independent predictors of survival in advanced nasopharyngeal carcinoma. J Transl Med, 2008, 6: 1

42 Pan J, Kong L, Lin S, et al. The clinical significance of coexpression of cyclooxygenases-2, vascular endothelial growth factors, and epidermal growth factor receptor in nasopharyngeal carcinoma. Laryngoscope, 2008, 118: 1970-1975

43 Zeng Z Y, Zhou Y H, Zhang W L, et al. Gene expression profiling of nasopharyngeal carcinoma reveals the abnormally regulated Wnt signaling pathway. Hum Pathol, 2007, 38: 120-133

44 Zhang L M, Deng T, Li X Y, et al. MicroRNA-141 is involved in a nasopharyngeal carcinoma-related genes network. Carcinogenesis, 2010, 31: 559-566

45 Zhou H D, Li X L, Li G Y, et al. Effect of SPLUNC1 protein on the Pseudomonas aeruginosa and Epstein-Barr virus. Mol Cell Biochem, 2008, 309: 191-197
46 Nakada C, Matsuura K, Tsukamoto Y, et al. Genome-wide microRNA expression profiling in renal cell carcinoma: Significant down-regulation of miR-141 and miR-200c. J Pathol, 2008, 216: 418-427

47 Xiao B, Fan S, Zeng Z, et al. Purification of novel UBAP1 protein and its decreased expression on nasopharyngeal carcinoma tissue microarray. Protein Expr Purif, 2006, 47: 60-67

48 Chalhoub N, Baker S J. PTEN and the PI3-kinase pathway in cancer. Annu Rev Pathol, 2009, 4: 127-150

49 Shen G P, Pan Q H, Hong M H, et al. Human genetic variants of homologous recombination repair genes first found to be associated with Epstein-Barr virus antibody titers in healthy cantonese. Int $\mathbf{J}$ Cancer, 2010, doi: 10.1002/ijc.25759

50 Ran Y, Wu S, You Y. Demethylation of E-cadherin gene in nasopharyngeal carcinoma could serve as a potential therapeutic strategy. J Biochem, 2010, doi: 10.1093/jb/mvq128

51 Guo Y, Chen J X, Yang S, et al. Selection of reliable reference genes for gene expression study in nasopharyngeal carcinoma. Acta Pharmacol Sin, 2010, 31: 1487-1494

$52 \mathrm{Xu} \mathrm{Y} \mathrm{F,} \mathrm{Liu} \mathrm{W} \mathrm{L,} \mathrm{Dong} \mathrm{J} \mathrm{Q,} \mathrm{et} \mathrm{al.} \mathrm{Sequencing} \mathrm{of} \mathrm{DC-SIGN} \mathrm{promoter}$ indicates an association between promoter variation and risk of nasopharyngeal carcinoma in cantonese. BMC Med Genet, 2010, doi: 10.1186/1471-2350-11-161

53 Wang S, Xiao X, Zhou X, et al. TFPI-2 is a putative tumor suppressor gene frequently inactivated by promoter hypermethylation in nasopharyngeal carcinoma. BMC Cancer, 2010, doi: 10.1186/14712407-10-617

54 Strebhardt K, Ullrich A. Targeting Polo-like kinase 1 for cancer therapy. Nat Rev Cancer, 2006, 6: 321-330

55 Wei S, Nehad M A, Carlo B, et al. Significance of Plk1 regulation by miR-100 in human nasopharyngeal cancer. Int J Cancer, 2010, 126: 2036-2048

56 Liu X, Lei M, Erikson R L. Normal cells, but not cancer cells, survive severe Plk1 depletion. Mol Cell Biol, 2006, 26: 2093-2108

57 Jang Y J, Ji J H, Choi Y C, et al. Regulation of Polo-like kinase 1 by DNA damage in mitosis: Inhibition of mitotic PLK-1 by protein phosphatase 2A. J Biol Chem, 2007, 282: 2473-2482

58 Sun F, Mikuni S, Kinjo M. Monitoring the caspase cascade in single apoptotic cells using a three-color fluorescent protein substrate. Biochem Biophys Res Commun, 2010, doi: 10.1016/j.bbrc.2010. 12.047

59 Syljuasen R G, Jensen S, Bartek J, et al. Adaptation to the ionizing radiation-induced $\mathrm{G} 2$ checkpoint occurs in human cells and depends on checkpoint kinase 1 and Polo-like kinase 1 kinases. Cancer Res, 2006, 66: 10253-10257

60 Spankuch B, Kurunci-Csacsko E, Kaufmann M, et al. Rational combinations of siRNAs targeting Plk1 with breast cancer drugs. Oncogene, 2007, 26: 5793-5807

61 Su S F, Han F, Zhao C, et al. Long-term outcomes of early-stage nasopharyngeal carcinoma patients treated with intensity-modulated radiotherapy alone. Int J Radiat Oncol Biol Phys, 2010, doi: 10.1016/ j.ijrobp.2010.09.011

62 Zhao L, Wan Q, Zhou Y, et al. The role of replanning in fractionated intensity modulated radiotherapy for nasopharyngeal carcinoma. Radiother Oncol, 2010, doi: 10.1016/j.radonc.2010.10.009

63 Shai E, Varon D. Development, cell differentiation, angiogenesisMicroparticles and their roles in angiogenesis. Arterioscler Thromb Vasc Biol, 2011, 31: 10-14

64 Lv X, Xiang Y Q, Cao S M, et al. Prospective validation of the prognostic value of elevated serum vascular endothelial growth factor in patients with nasopharyngeal carcinoma: More distant metastases and shorter overall survival after treatment. Head Neck, 2010, doi: 10.1002/hed.21541

65 Hua Z, Lv Q, Ye W B, et al. MiRNA-directed regulation of VEGF and other angiogenic factors under hypoxia. PLoS One, 2006, 1: e116

66 Olsson A K, Dimberg A, Kreuger J, et al. VEGF receptor signalling-in control of vascular function. Nat Rev Mol Cell Biol, 2006, 7: $359-371$

67 Li G, Wu Z R, Peng Y, et al. MicroRNA-10b induced by Epstein-Barr virus-encoded latent membrane protein-1 promotes the metastasis of 
human nasopharyngeal carcinoma cells. Cancer Lett, 2010, 299: 29-36

68 Chew M M, Gan S Y, Khoo A S, et al. Interleukins, laminin and Epstein-Barr virus latent membrane protein 1 (EBV LMP1) promote metastatic phenotype in nasopharyngeal carcinoma. BMC Cancer, 2010, 10: 574

69 Kim T J, Lee Y S, Kang J H, et al. Prognostic significance of expression of vegf and cox-2 in nasopharyngeal carcinoma and its association with expression of C-erbB2 and EGFR. J Surg Oncol, 2010, doi: $10.1002 /$ jso. 21767

70 Sikumar S, Johan A B, Chen I H, et al. MicroRNA 29c is down-regu- lated in nasopharyngeal carcinomas, up-regulating mRNAs encoding extracelluar matrix proteins. Proc Natl Acad Sci USA, 2008, 105: 5874 5878

71 Xia H, Ng S S, Jiang S, et al. MiR-200a-mediated downregulation of ZEB2 and CTNNB1 differentially inhibits nasopharyngeal carcinoma cell growth, migration and invasion. Biochem Biophys Res Commun, 2010, 391: 535-541

72 Chen L C, Chen C C, Liang Y, et al. A novel role for TNFAIP2: Its correlation with invasion and metastasis in nasopharyngeal carcinoma. Mod Pathol, 2010, doi: 10.1038/modpathol.2010.193

Open Access This article is distributed under the terms of the Creative Commons Attribution License which permits any use, distribution, and reproduction in any medium, provided the original author(s) and source are credited. 